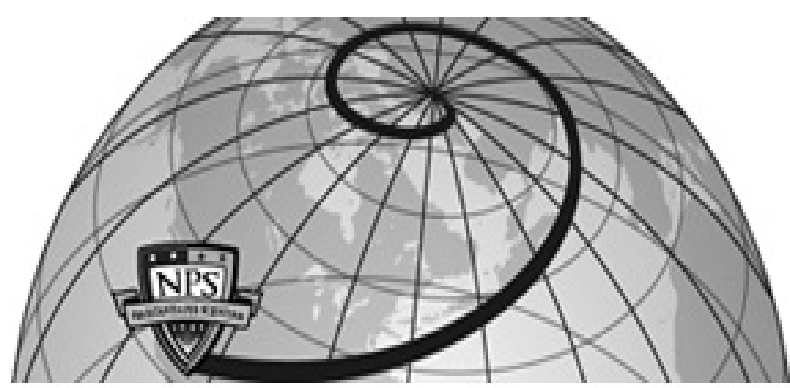

Calhoun: The NPS Institutional Archive DSpace Repository

\title{
Improving Localization Accuracy using Spatio-Temporal Information of Objects
}

Rowe, Neil C.; Chen, Xiao; Singh, Gurminder

Monterey, California. Naval Postgraduate School

Journal of Parallel and Distributed Computing, Vol. 72, Issue 8, August 2012 https://hdl.handle.net/10945/35968

This publication is a work of the U.S. Government as defined in Title 17, United States Code, Section 101. Copyright protection is not available for this work in the United States.

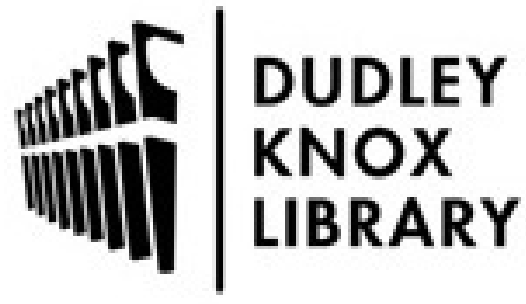

http://www.nps.edu/library
Calhoun is the Naval Postgraduate School's public access digital repository for research materials and institutional publications created by the NPS community. Calhoun is named for Professor of Mathematics Guy K. Calhoun, NPS's first appointed -- and published -- scholarly author.

Dudley Knox Library / Naval Postgraduate School 411 Dyer Road / 1 University Circle Monterey, California USA 93943 


\section{Improving Localization Accuracy using Spatio-Temporal Information of Objects}

\author{
Xiao Chen \\ Dept. of Computer Science \\ Texas State University \\ San Marcos, TX 78666 \\ Email: xc10@txstate.edu
}

\author{
Neil C. Rowe \\ and Gurminder Singh \\ Dept. of Computer Science \\ U. S. Naval Postgraduate School \\ Monterey, CA 93943 \\ Email: ncrowe@nps.edu
}

\begin{abstract}
Improvised explosive devices (IEDs) are an increasingly serious military threat as is witnessed in Iraq and Afghanistan. To combat the IED emplacement, it is important to have persistent surveillance over time. Due to the low cost and capabilities of sensors, wireless sensor networks (WSNs) have tremendous potential for military and civilian surveillance. In this paper, we explore methods to improve an important aspect of surveillance: localization accuracy. Though there are many localization algorithms in the literature, all of them try to improve the accuracy from the side of sensor networks. In this paper, we tackle this problem from a new angle, that is, we look at the spatio-temporal relationships of objects we track, which, as far as we know, unprecedented in this attempt. We first develop algorithms that use spatial and temporal relationships of objects separately and then design ones that combine them. Experimental results show that all our proposed algorithms can improve localization accuracy, especially those combined ones. Moreover, since our methods use features related to objects themselves and not the underlying localization mechanism, they can be built on any localization algorithm to improve accuracy.
\end{abstract}

\section{INTRODUCTION}

Improvised explosive devices (IEDs) are an increasingly serious military threat. Detection of emplaced IEDs is very difficult as is witnessed recently in Iraq and Afghanistan. To combat the IED emplacement, it is important to have persistent surveillance over time [14]. Due to the low cost of sensors and their capabilities that can avoid occlusion and confusion in depth, can violate privacy less of those tracked, can be easier to conceal from adversary countermeasures, and can be distributed over large areas to provide uniform coverage, wireless sensor networks (WSNs) have tremendous potential in pervasive surveillance in military and civilian contexts. To realize such a system, in our previous work [13], we have explored the capabilities and limitations of sensor networks to track objects' locations, which is an important aspect of surveillance. In this paper, we plan to investigate methods to improve localization accuracy by taking the spatio-temporal relationships of objects into account.

To tackle the localization problem, in the literature, there are range-based methods [1], [3], [10]-[12], [16] and rangefree methods [5], [7], [8]. And more recently to save energy of WSNs, sensors to monitor objects are activated dynamically. There are collaborative approaches [15] and prediction-based techniques [2], [6].
Although these methods can improve localization accuracy by using better algorithms on the side of sensor networks, none of the previous work has thought about using the spatiotemporal relationships of objects themselves. As we know, to attain their goals, malicious people rarely act in individuals. They usually have a team and collaborate with each other in actions such as IED emplacement. Besides, acting in a group can be more likely to confuse the surveillance system, especially if they dress and behave similarly. In nature, fish swim in schools to avoid sharks and birds fly in flocks to avoid hawks, because in this way it is very difficult for predators to track each individual for sufficient amount of time to catch it. On the other hand, from the point of localization, if they act in a group, their spatial relationship gives us an extra condition to locate them better. Similarly, if we know how an object moves over a period of time, that is, the temporal relationship in its footsteps, we can also locate it better. In this paper, we are interested in exploring methods to improve localization accuracy using spatio-temporal relationships of objects. Networking issues are not considered because they are not our main focus here.

The main contributions of this paper are: (1) we put forward methods to improve localization accuracy using spatiotemporal relationships among objects themselves, which, to our knowledge, unprecedented in this endeavor. (2) we conduct simulations to validate the effectiveness of our methods. Experimental results show that all our proposed algorithms can improve localization accuracy, especially those combing both spatial and temporal information. (3) because our methods use the features related to objects themselves, and not the underlying mechanism to locate them, they can be built on any localization algorithm to improve localization accuracy.

The remainder of this paper is organized as follows: Section II mentions the related work. Section III formulates the problem of using spatio-temporal relationships among objects to improve localization accuracy. Section IV-A, IV-B, IV-C, and IV-D put forward methods to achieve that. Section V shows the simulation results of the proposed approaches. And finally, Section VI draws the conclusion. 


\section{RELATED WORK}

In the literature, there are range-based and range-free localization methods. The range-based methods depend on the assumption that the absolute distance between a sender and a receiver can be estimated by one or more features of the communication signal from the sender to the receiver. The features of the communication signal that are frequently used include angle of arrival [10], [11], signal strength [1], [12] and time of arrival/time difference of arrival [3], [12], [16]. Range-free methods [5], [7], [8] depend on the content of the received message and no special hardware and the above range measurements are used.

More recently, in order to save more energy of WSNs, sensors are activated dynamically and work collaboratively to monitor objects. The examples are cluster-based scheme [15], in which sensor nodes are dynamically clustered based on received signal strength to collaboratively track single target; and prediction-based techniques [2], [6], which predict the future movement of mobile targets so as to turn on only those sensors necessary to sense targets in order to save energy of WSNs.

\section{PROBLEM FORMULATION}

Though the above methods can improve localization accuracy by using better algorithms in WSNs, none of them has considered improving the accuracy using the features of objects being monitored.

In this paper, we assume that some sensor nodes are deployed over an area to track moving objects that intrude the monitored region. We also assume that there is a central site that collects the sensor readings about the target, based on which to estimate its location. How the sensors send the data and the frequency to send the data to the central site are important areas of research in sensor applications, but out of scope of our study. Here, we just focus on methods to improve localization accuracy. To locate objects, some localization algorithm in the literature can be used. The accuracy of localization depends on the quality of the localization algorithm. Now we further assume that objects move coherently in a group and they move in a certain direction with a certain speed over time. When objects move in a group, their relative locations create the spatial relationship among them. And when an object moves along a certain direction with a certain speed, its footsteps in the trajectory, which are the locations of the object at different times, are temporally related. Now the problem is formulated as: given the estimated locations of objects by some regular localization algorithm, is it possible to improve localization accuracy through the spatio-temporal relationships of objects? This is the problem that we want to explore in this paper.

\section{LOCALIZATION ACCURACY IMPROVEMENT ALGORITHMS}

In this section, we present our various localization accuracy improvement algorithms.

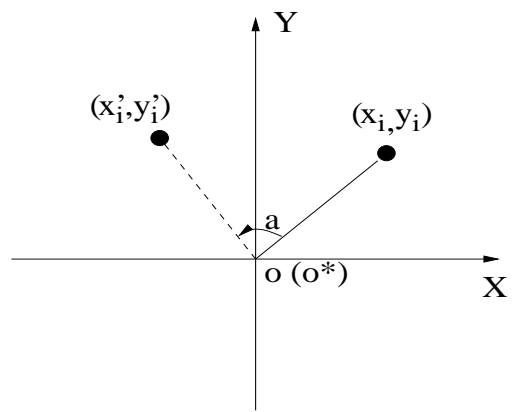

Fig. 1. An object's location relative to the centroid of the group before and after the movement

\section{A. Improve Localization Accuracy by Space}

In this subsection, we put forward an algorithm to improve localization accuracy using the spatial relationship of objects.

Suppose there are $n$ objects moving coherently in a group and the estimated locations of these objects calculated by some localization algorithm are: $u_{1}^{*}, u_{2}^{*}, \cdots, u_{n}^{*}$. Suppose initially the relative location of an object $u_{i}(1 \leq i \leq n)$ to the group's centroid $o$ is $\left(x_{i}, y_{i}\right)$. When the group moves to a certain location in the sensor field, the relative location of node $u_{i}$ to the group's centroid $o^{*}$ is $\left(x_{i}^{\prime}, y_{i}^{\prime}\right)$. If we overlap $o$ with $o^{*}$ (see Figure 1), the relationship between $\left(x_{i}, y_{i}\right)$ and $\left(x_{i}^{\prime}, y_{i}^{\prime}\right)$ can be expressed as:

$$
\left[\begin{array}{l}
x_{i}^{\prime} \\
y_{i}^{\prime}
\end{array}\right]=\left[\begin{array}{ll}
r & 0 \\
0 & r
\end{array}\right]\left[\begin{array}{cc}
\cos \alpha & -\sin \alpha \\
\sin \alpha & \cos \alpha
\end{array}\right]\left[\begin{array}{l}
x_{i} \\
y_{i}
\end{array}\right]
$$

Here, $\alpha$ is an angle turned after the group moves to a certain location and $r$ is an adjustment parameter. From the estimated values: $u_{1}^{*}, u_{2}^{*}, \cdots, u_{n}^{*}$, we calculate the centroid $o^{*}$ of the group and the relative position of each object $\left(x_{i}^{*}, y_{i}^{*}\right)$ to their centroid $o^{*}$ as follows:

$$
\begin{cases}o_{x}^{*}=\frac{\sum_{i=1}^{n} u_{i x}^{*}}{n}, & x_{i}^{*}=u_{i x}^{*}-o_{x}^{*}, \\ o_{y}^{*}=\frac{\sum_{i=1}^{n} u_{i y}^{*}}{n}, & y_{i}^{*}=u_{i y}^{*}-o_{y}^{*} .\end{cases}
$$

Now we want to minimize the error term between our calculated relative locations of all objects $\left(x_{i}^{\prime}, y_{i}^{\prime}\right)(1 \leq i \leq n)$ and the estimated relative locations by some localization algorithm of all objects $\left(x_{i}^{*}, y_{i}^{*}\right)(1 \leq i \leq n)$. So it is to minimize function:

$$
f(\alpha, r)=\sum_{i=1}^{n}\left[\left(x_{i}^{\prime}-x_{i}^{*}\right)^{2}+\left(y_{i}^{\prime}-y_{i}^{*}\right)^{2}\right]
$$

To do that, the partial derivatives should be equal to 0 . After solving equations, $\alpha$ and $r$ are as follows:

$$
\left\{\begin{array}{l}
\alpha=\arctan \frac{\sum_{i=1}^{n}\left(x_{i} y_{i}^{*}-x_{i}^{*} y_{i}\right)}{\sum_{i=1}^{n}\left(x_{i} x_{i}^{*}+y_{i} y_{i}^{*}\right)}, \\
r=\frac{\sum_{i=1}^{n}\left[\left(x_{i} x_{i}^{*}+y_{i} y_{i}^{*}\right) \cos \alpha+\left(x_{i} y_{i}^{*}-x_{i}^{*} y_{i}\right) \sin \alpha\right]}{\sum_{i=1}^{n}\left(x_{i}^{2}+y_{i}^{2}\right)} .
\end{array}\right.
$$

Knowing $\alpha$ and $r$, the adjusted location of each object $\left(x_{i}^{\prime}, y_{i}^{\prime}\right)$ relative to centroid $o^{*}$ can be calculated by Eq. (1). Thus, the location $u_{i}^{\prime}$ in the network can be adjusted to: 


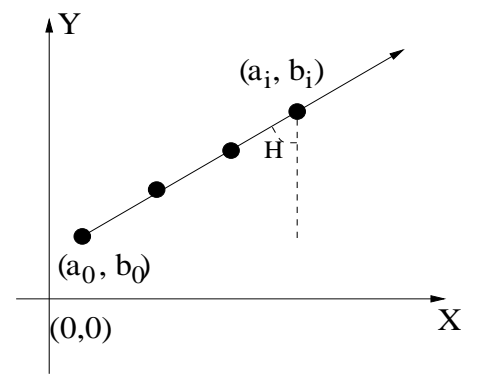

Fig. 2. An object's trajectory over a sensor field

$$
\begin{aligned}
& {\left[\begin{array}{l}
u_{i x}^{\prime} \\
u_{i y}^{\prime}
\end{array}\right]=\left[\begin{array}{l}
o_{x}^{*} \\
o_{y}^{*}
\end{array}\right]+\left[\begin{array}{l}
x_{i}^{\prime} \\
y_{i}^{\prime}
\end{array}\right]} \\
& =\left[\begin{array}{c}
o_{x}^{*} \\
o_{y}^{*}
\end{array}\right]+r\left[\begin{array}{l}
x_{i} \cos \alpha-y_{i} \sin \alpha \\
x_{i} \sin \alpha+y_{i} \cos \alpha
\end{array}\right]
\end{aligned}
$$

\section{B. Improve Localization Accuracy by Time}

In this subsection, we improve localization accuracy by looking at the footsteps of an object over time. Suppose we know that an object travels along a line with a certain speed and the estimated locations of this object using some localization method from time 1 to $t$ are: $\left(a_{1}^{*}, b_{1}^{*}\right),\left(a_{2}^{*}, b_{2}^{*}\right), \cdots,\left(a_{t}^{*}, b_{t}^{*}\right)$. Here, time 1 is not necessarily the time when the object starts moving. It is the time during its movement that we start to observe. The problem is to adjust these estimated footsteps to make them closer to the actual locations. We explore methods in two conditions: (1) the speed and direction of the object are known and (2) the speed and direction of the object are unknown.

1) Speed and direction are known: Suppose the speed of the object is $v$ and the direction angle is $H$ (see Figure 2), the starting point of this object, which is unknown, is $\left(a_{0}, b_{0}\right)$, then the location of this object at time $i(1 \leq i \leq t)$ should be: $a_{i}=a_{0}+i v \sin (H), b_{i}=b_{0}+i v \cos (H)$.

Now our task is to reduce the error term between the calculated locations and the estimated locations. So it is to minimize function:

$$
\begin{aligned}
& f\left(a_{0}, b_{0}\right)=\sum_{i=1}^{t}\left[\left(a_{i}-a_{i}^{*}\right)^{2}+\left(b_{i}-b_{i}^{*}\right)^{2}\right] \\
= & \left.\sum_{i=1}^{t}\left[a_{0}+i v \sin (H)-a_{i}^{*}\right)^{2}+\left(b_{0}+i v \cos (H)-b_{i}^{*}\right)^{2}\right]
\end{aligned}
$$

To do that, the partial derivatives should be equal to zero. Thus, $a_{0}$ and $b_{0}$ can be found as:

$$
\left\{\begin{array}{l}
a_{0}=\frac{1}{t} \sum_{i=1}^{t} a_{i}^{*}-\frac{t+1}{2} v \sin (H), \\
b_{0}=\frac{1}{t} \sum_{i=1}^{t} b_{i}^{*}-\frac{t+1}{2} v \cos (H) .
\end{array}\right.
$$

Next, each estimated location $\left(a_{i}^{*}, b_{i}^{*}\right)$ can be adjusted to $\left(a_{i}^{\prime}, b_{i}^{\prime}\right)$ as follows:

$$
\left[\begin{array}{c}
a_{i}^{\prime} \\
b_{i}^{\prime}
\end{array}\right]=\left[\begin{array}{c}
a_{0}+i v \sin (H) \\
b_{0}+i v \cos (H)
\end{array}\right]
$$

2) Speed and direction are unknown: Suppose the unknown speed of the object is $v_{x}$ in $x$ direction and $v_{y}$ in $y$ direction and the starting point of this object, which is also unknown, is $\left(a_{0}, b_{0}\right)$, then the location of this object at time $i(1 \leq i \leq t)$ should be: $a_{i}=a_{0}+i v_{x}, b_{i}=b_{0}+i v_{y}$.

Now our task is to reduce the error term between the calculated locations and the estimated locations. So it is to

$$
\begin{aligned}
& f\left(v_{x}, v_{y}, a_{0}, b_{0}\right)=\sum_{i=1}^{t}\left[\left(a_{i}-a_{i}^{*}\right)^{2}+\left(b_{i}-b_{i}^{*}\right)^{2}\right] \\
& \left.=\sum_{i=1}^{t}\left[a_{0}+i v_{x}-a_{i}^{*}\right)^{2}+\left(b_{0}+i v_{y}-b_{i}^{*}\right)^{2}\right]
\end{aligned}
$$

To do that, the partial derivatives should be equal to zero. Thus, $v_{x}, v_{y}, a_{0}$ and $b_{0}$ can be found as:

$\begin{cases}v_{x}=\frac{4 \sum_{i=1}^{t} i a_{i}^{*}-2(n+1) \sum_{i=1}^{t} a_{i}^{*}}{4 \sum_{i=1}^{t} i^{2}-n(n+1)^{2}}, & a_{0}=\frac{1}{n} \sum_{i=1}^{t} a_{i}^{*}-\frac{n+1}{2} v_{x}, \\ v_{y}=\frac{4 \sum_{i=1}^{t} i b_{i}^{*}-2(n+1) \sum_{i=1}^{t} b_{i}^{*}}{4 \sum_{i=1}^{t} i^{2}-n(n+1)^{2}}, & b_{0}=\frac{1}{n} \sum_{i=1}^{t} b_{i}^{*}-\frac{n+1}{2} v_{y} .\end{cases}$

Next, each estimated location $\left(a_{i}^{*}, b_{i}^{*}\right)$ can be adjusted to $\left(a_{i}^{\prime}, b_{i}^{\prime}\right)$ as follows:

$$
\left[\begin{array}{c}
a_{i}^{\prime} \\
b_{i}^{\prime}
\end{array}\right]=\left[\begin{array}{c}
a_{0}+i v_{x} \\
b_{0}+i v_{y}
\end{array}\right]
$$

\section{Improve Localization Accuracy by Space and Time}

In this subsection, we combine the spatial and the temporal information. First we use the spatial information and then use the temporal information. If we know the estimated locations of all group members over the past $t$ time units, we first use Eq. (2) to adjust their estimated locations using spatial information in the group and then use Eq. (3) or Eq. (4) to adjust each object according to its footsteps in the past $t$ time units.

\section{Improve Localization Accuracy by Time and Space}

This subsection is the reverse of the previous subsection, we first apply the temporal information and then the spatial information. Suppose we know the estimated locations of all group members over the past $t$ time units, we first use Eq. (3) or Eq. (4) to adjust each object according to its footsteps in the past $t$ time units and then apply Eq. (2) to adjust the locations of all objects using the spatial information in the group.

\section{Simulations}

In this section, we conduct simulations to see how our algorithms can improve the accuracy of localization. We build a simulator in the Matlab language. We can start from any localization algorithm in the literature and apply spatio-temporal relationships of objects to improve localization accuracy. In this paper, we adopt the localization method by signal strength in our previous work [13] as a foundation. We call this method our original method (ORG), and from it, we apply LAS, LAT1, LAT2, LAST and LATS listed below. Here when we combine time and space, we use LAT1 as an example. Thus, we compare the following six methods: 


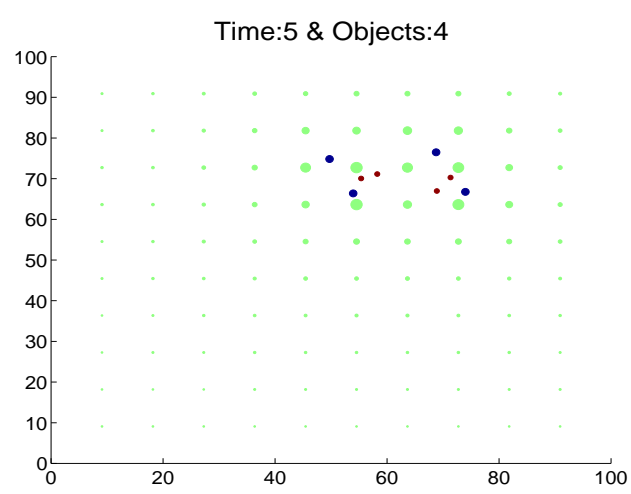

(a) Snapshot using ORG

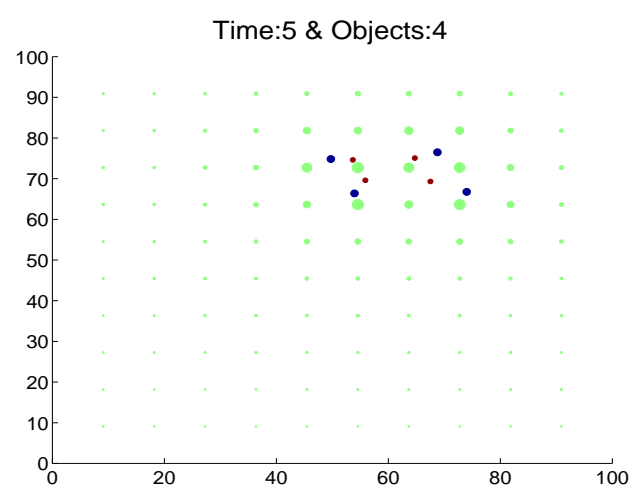

(b) Snapshot using LAST

Fig. 3. Snapshots using ORG and LAST localization algorithms

1) original localization algorithm (ORG)

2) localization using space (LAS)

3) localization using time with known speed and direction (LAT1)

4) localization using time with unknown speed and direction (LAT2)

5) localization using space first then time (LAST)

6) localization using time first then space (LATS)

To make the paper self-inclusive, we introduce the main idea of using signal strength to track objects in paper [13] here. A grid of $100 \times 100$ sensors are deployed in the field (see green dots in Figure 3). Random objects are created and signals from these sources are received by the sensors in the field. Following discussions of acoustic, seismic and magnetic signals in the literature [9], the inverse-square law is a good model to calculate signal strength, where each signal strength is:

$$
s_{i}=c+\frac{a}{(m+d)^{2}}, \quad 1 \leq i \leq N
$$

Here, $s_{i}$ is the sensed signal strength in the $i$ th sensor, $c$ is a random factor, $a$ is the intensity of the source, $d$ is the distance from the source to the sensor, $m$ is the minimumdistance factor from the source which is a feature of each sensor and $N$ is the total number of sensors. Parameter $c$ is set to 0 and to avoid unstable behavior with very-near sources, $m$ is set to 5 based on experiments in [13]. The total signal strength received by each sensor is assumed to be additive from all these sources.

Paper [13] estimates the locations of objects in two steps. First, estimate where objects are as the places of the nearest sensors that receive the maximum signal strength in their neighborhood (local maxima). The neighborhood of a sensor includes all the sensors that are one grid space away from the current one. For example, for an internal sensor on the grid, it has four neighbors one grid space away in the east, south, west, and north directions. Second, improve the localization accuracy based on observed ratio of signal strengths, a variation of the approach in [9]. If initially the observed signal strength is assumed only due to each sensor's nearest source based on the fact that the effects fall off fast with an inverse square, then for two sensors 1 and 2 nearest the same signal, the following holds true:

$$
s_{1}\left[\left(x-x_{1 s}\right)^{2}+\left(y-y_{1 s}\right)^{2}+m^{2}\right]=s_{2}\left[\left(x-x_{2 s}\right)^{2}+\left(y-y_{2 s}\right)^{2}+m^{2}\right]
$$

Here, $s_{1}$ and $s_{2}$ represent the signal strength received by the two sensors, $m$ represents the minimum-distance factor as mentioned above, $(x, y)$ is the position of the tracked object, and $\left(x_{1 s}, y_{1 s}\right)$ and $\left(x_{2 s}, y_{2 s}\right)$ are the coordinates of the two sensors. Rearranging this gives an equation of a circle for the locus of points on which the sensor could lie. The center and radius of this circle are:

$$
\begin{gathered}
x_{c}=\frac{s_{1} x_{1}-s_{2} x_{2}}{s_{1}-s_{2}}, \quad y_{c}=\frac{s_{1} y_{1}-s_{2} y_{2}}{s_{1}-s_{2}} \\
r=\sqrt{\frac{s_{1} s_{2}\left[\left(x_{1 s}-x_{2 s}\right)^{2}+\left(y_{1 s}-y_{2 s}\right)^{2}\right]}{\left(s_{1}-s_{2}\right)^{2}}-m^{2}}
\end{gathered}
$$

Next, use the idea of trilateration [4] to locate the sources. From each nearest sensor inferred from step one, find its neighboring sensors. If there are two neighbors, two circles can be formed by the nearest sensor with each neighbor respectively as above. The two circles can reduce the locus to two points. If there are more than two neighbors, a consensus center can be obtained by finding the set of all intersection points and repeatedly removing the furthest point from the centroid of the set until only two points remaining. The centroid of these two points is the inferred source location from Step two. Once all source locations are inferred like this, the signal strength of the sensors can be computed, then the source locations can be recomputed iteratively until accuracy is sufficiently good. In [13], step two is repeated twice to reach a good accuracy, so iteration is not really necessary.

Based on these previous results, in this paper, we generate a group of $n$ objects randomly in a $100 \times 100$ grid. It travels in some direction with some speed across the grid. As it travels, the associated signal strengths received by sensors are calculated at evenly spaced time points to provide test cases for localization algorithms. The time starts from 1 and the interval between time points is one second. The calculation stops if one of the objects flies out of the grid boundary. Next we infer object locations from the signal strength patterns using 

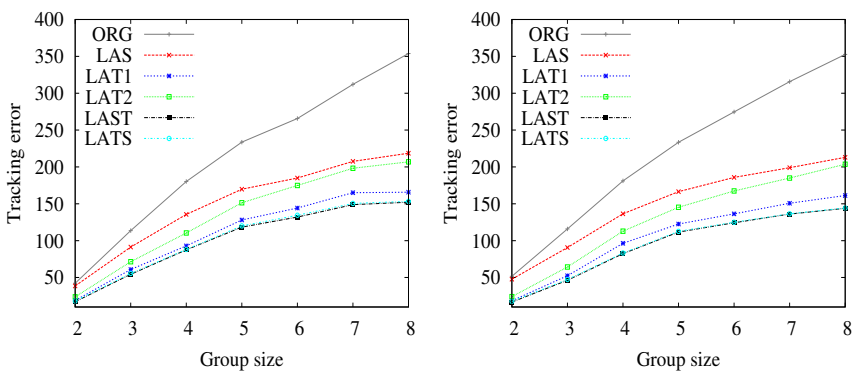

(a) Tracking error in different group (b) Tracking error in different group size with variance 1 size with variance 3

Fig. 4. Tracking error with different group sizes and variances

the ORG algorithm. After that, by considering spatio-temporal relationships of objects, we apply LAS, LAT1, LAT2, LAST, and LATS independently to obtain the adjusted locations of objects at each second. For example, Figures 3(a) and 3(b) show the snapshots of tracking simulator with a group of 4 objects at time 5 using ORG and LAST algorithms. The snapshots of other algorithms are not shown here because of space limit. In the figures, the blue dots are the actual locations of the objects. The green dots represent the sensors. The size of a green dot shows the signal strength received by the sensor. The larger the size, the stronger the signal. The purple dots represent the estimated locations by one of the six algorithms. The closer the purple dots to the blue dots, the more accurate the estimation. Once the locations of objects are estimated, they can be used as inputs for the next round of calculation. In other words, each algorithm can be called repeated until accuracy is good enough. But we find that the first call does the major adjustment, so we do not pursue accuracy further by iteration.

We use metric tracking error to measure the tracking error of these algorithms. Metric tracking error $=\sum_{i=1}^{n}\left(\left|u_{i}^{*} u_{i}\right|^{2}\right)$. $\left|u_{i}^{*} u_{i}\right|^{2}$ is the distance square of a node's estimated location $u_{i}^{*}$ to its actual location $u_{i}$. Tracking error here is defined as the summation of tracking errors of all the objects. So the more the objects, the larger the tracking error. We try groups with 2 , $3, \cdots$, and 8 objects and with signal variances 1 and 3 . Signal variance is the error in the signal strength perceived by the sensor. For each entry, we run 100 times and the tracking error is averaged over these runs. Figures 4(a) and 4(b) show the results. All the algorithms put forward in this paper outperform the ORG algorithm. The LAS algorithm is better than ORG, LAT2 is better than LAS, LAT1 is better than LAT2, and LAST and LATS are better than LAT2. LAT1 outperforms LAT2 because if more parameters are known, the results can be more accurate. The combination of space and time is better than each individual one. But there is not much difference between LAST and LATS as their curves are overlapped. In other words, whether the locations are adjusted by space first or by time first does not matter much. Also, the signal variance has little effect on tracking error as the graphs look almost the same. To conclude, adding spatio-temporal relationships of objects can improve localization accuracy.

\section{CONCLUSION}

In this paper, we designed algorithms to improve localization accuracy by including spatio-temporal relationships among objects we track. We first considered the spatial relationship among objects when they move coherently in a group, then looked at the temporal relationship within an object's footsteps in its trajectory, and then tried the combination of the two. Simulation results showed that all our algorithms can improve localization accuracy, especially those combined ones. In the future, we will apply these methods on actual sensors such as acoustic, infrared, and seismic sensors in the field to see how they can help to track suspicious objects to combat the IED emplacement.

\section{ACKNOWLEDGMENTS}

This research was supported in part by NSF grant CBET 0729696.

\section{REFERENCES}

[1] P. Bahl and V. N. Padmanabhan, "RADAR: An in-building RF-based user location and tracking system," Proc. of the IEEE INFOCOM'OO, Mar. 2000, pp. 775-784.

[2] M. Z. A. Bhuiyan, G. Wang, and J. Wu, "Target tracking with monitor and backup sensors in wireless sensor networks," Proc. of the IEEE ICCCN'09, Aug. 2009.

[3] C. R. Comsa, J. H. Luo, A. Haimovich, S. Schwartz, "Wireless localization using time difference of arrival in narrow-band multipath systems," Proc. of International Symposium on Signals, Circuits and Systems, vol. 2, July 2007, pp. 1-4.

[4] J. Figueiras, H. P. Schwefel, and I. Kovacs, "Accuracy and timing aspects of location information based on signal-strength measurements in Bluetooth," Proc. of the 16th IEEE International Symposium on Personal, Indoor, and Mobile Radio Communications, Sept. 2005, pp. 2685-2690.

[5] S. Guha, R. Murty, and E. Sirer, "Sextant: a unified node and event localization framework using non-convex constraints," Proc. of ACM International Symposium on Mobile Ad Hoc Networking and Computing (MobiHoc), 2005, pp. 205-216.

[6] Z. Guo, M. Zhou and L. Zakrevski, "Optimal tracking interval for predictive tracking in wireless sensor network," IEEE Communication Letters, vol. 9, 2005, pp. 805-807.

[7] T. He, C. Huang, B. M. Blum, J. A. Stankovic, and T. F. Abdelzaher, "Range-free localization schemes in large scale sensor networks," Proc. of ACM MobiCom'03, 2003, pp. 81-95.

[8] B. Kusý, A. Lédeczi, M. Maróti, and L. Meertens, "Node density independent localization," Proc. of the fifth International Conference on Information Processing in Sensor Networks (IPSN)", 2006, pp. 441-448.

[9] J. Lee, K. Cho, S. Lee, T. Kwon, and Y. Choi, "Distributed and energyefficient target localization and tracking in wireless sensor networks," Computer Communications, vol. 29, 2006, pp. 2494-2505.

[10] A. Nasipuri and K. Li, "A directionality based location discovery scheme for wireless sensor networks," Proc. of ACM WSNA'02, Sept. 2002.

[11] D. Niculescu and B. Nath, "Ad hoc positioning system (APS) using AoA," Proc. of IEEE INFOCOM'03, 2003, pp. 1734-1743.

[12] N. Patwari, A. O. Hero, M. Perkins, N. S. Correal, and R. J. ODea, "Relatvie location estimation in wireless sensor networks," IEEE Transactions on Signal Processing, vol. 51, no. 8, Aug. 2003.

[13] N. Rowe, M. O'Hara, and G. Singh, "Wireless sensor networks for detection of IED emplacement," Proc. of Intl. Command and Control Research and Technology Symposium, Washington, DC, June 2009.

[14] W. Saletan, "The Jihadsons: technology lessons from the Iraq war," Slate Magazine, Oct. 12, 2007, retrieved Sept. 2008 from www.slate.com/id/2175723.

[15] S. Suganya, "A cluster-based approach for collaborative target tracking in wireless sensor networks," Proc. of the 1st Inernational Conference on Emerging Trends in Engineering and Technology (ICETET 2008), 2008, pp. 276-281.

[16] B. H. Wellenhoff, H. Lichtenegger and J. Collins, "Global positions system: theory and practice," Fourth Edition, Springer Verlage, 1997. 